\title{
Reflection and transmission of waves from imperfect boundary between two heat conducting micropolar thermoelastic solids
}

\author{
Kunal Sharma and Marin Marin
}

\begin{abstract}
The problem of reflection and transmission of plane waves at an imperfect boundary between two thermally conducting micropolar elastic solid half spaces with two temperature is investigated. Amplitude ratio of various reflected and transmitted waves are presented when a set of coupled longitudinal wave (LD-wave) and thermal wave (T-wave) and a set of coupled transverse wave and microrotation waves (CDI, CDII) is made incident. The expressions for reflection and transmission coefficients which are the ratios of the amplitudes of reflected and transmitted waves at different angles of incident wave are obtained. The corresponding expressions for the normal force stiffness, transverse force stiffness, transverse couple stiffness and perfect bonding has also been included. Stiffness and two temperature effects on these amplitude ratios with angle of incidence have been depicted graphically. Some special and particular cases are also discussed.
\end{abstract}

\section{Introduction}

The exact nature of layers beneath the earths surface are unknown. Therefore, one has to consider various appropriate models for the purpose of theoretical

Key Words: Micropolar thermoelastic with two temperature, Reflection and transmission coefficient, Amplitude ratios, Normal force stiffness, Thermal conductness, Transverse force stiffness.

2010 Mathematics Subject Classification: Primary 74A15; Secondary 35Q79; 74J05; $80 \mathrm{~A} 20$.

Received: June, 2013

Accepted: August, 2013 
investigation. Modern engineering structures are often made up of materials possessing an internal structure. Polycrystalline materials, materials with fibrous or coarse grain structure come in this category. Classical elasticity is inadequate to represent the behaviour of such materials. The analysis of such materials requires incorporating the theory of oriented media. For this reason, micropolar theories were developed by Eringen[1,2,3] for elastic solids, fluids and further for non-local polar fields and are now universally accepted. A micropolar continuum is a collection of interconnected particles in the form of small rigid bodies undergoing both translational and rotational motions.

The linear theory of micropolar thermoelasticity was developed by extending the theory of micropolar continuum to include thermal effects by Eringen[4] and Nowacki[5]. Dost and Taborrok [6] presented the generalized thermoelasticity by using Green and Lindsay theory[7].

The main difference of thermoelasticity with two temperature with respect to the classical one is the thermal dependence. Chen et al [8,9] have formulated a theory of heat conduction in deformable bodies, which depend on two distinct temperature, the conductive temperature $\phi$ and thermodynamic temperature $\theta$. For time independent situations, the difference between these two temperature is proportional to the heat supply. For time dependent problems in wave propagation the two temperature are in general different. The two temperature and the strain are found to have representation in the form of a travelling wave pulse, a response which occurs instantaneously throughout the body Boley [10]. The wave propagation in the two temperature theory of thermoelasticity was investigated by Warren and Chen [11].

Various investigators Youssef [12] , Puri and Jordan [13], Youssef and Al-Lehaibi [14], Youssef and Al-Harby [15], Magana and Quintanilla [16], Mukhopadhyay and Kumar [17], Roushan and Santwana [18], Kaushal et al [19], Kaushal et al [20], Ezzat and Awad [21] and Ezzat et al. [22] studied different problems in thermoelastic medias with two temperature.

An actual interface between two elastic media is much more complicated and has physical properties different from those of the substrates. A generalization of this concept is that of an imperfectly bonded interface. In this case displacement across the surfaces need not be continuous. Imperfect bonding means that the stress components are continuous, but the displacement field is not. The small vector difference in the displacement is assumed to depend linearly on the traction vector. To describe the physical conditions on the interface by different mechanical boundary conditions, significant work has been done by different investigators. Notable among them are Baik and Thomson [23], Rokhlin [24], Angel and Achenbach [25], Pilarski and Rose [26], Lavrentyev and Rokhlin [27].

Recently various authors have used the imperfect conditions at an interface 
to study various types of problems e.g. Kumar and Sharma [28], Kumar etal. [29, 30, 31,32], Ram and Sharma [33], Kumar and Sharma [34], Sharma etal. [35], Kumar and Chawla [36, 37, 38].

The theory from [39] is a theory of thermoelasticity constructed by taking into account the heat conduction in deformable bodies which depends om two temperature.

In the context of in the paper [40] it is proved that the Cesaro means of the kinetic and strain energies of a solution with finite energy become asymptotic equal as time tends to infinity.

By using a measure of Toupin type associated with the corresponding steady-state vibration and assuming that the exciting frequency is lower than a certain critical frequency, in the paper [41] it is obtained a spatial decay estimate similar to that of Saint-Venant type.

In the paper [42] the author applies the theory of semigroups of operators in order to obtain the existence and uniqueness of solutions for the mixed initial-boundary value problems in thermoelasticity of dipolar bodies. The continuous dependence of the solutions upon initial data and supply terms is also proved

The reflection and transmission of plane waves i.e. Longitudinal displacement wave (LD wave), Thermal wave ( $\mathrm{T}$ wave), Coupled transverse wave (CD-I wave and CD-II wave) at an imperfect interface of two different micropolar generalized thermoelastic solid half spaces with two temperature has been investigated. Stiffness and two temperature effects are depicted graphically on the amplitude ratios for incidence of various plane waves.

\section{Basic equations}

Following Eringen [1], Warren and Chen [11] the field equations in an isotropic, homogeneous, micropolar elastic medium in the context of generalized theory of thermoelasticity with two temperature, without body forces, body couples and heat sources, are given by

$$
\begin{gathered}
(\alpha+\beta+\gamma) \nabla(\nabla \cdot \vec{\phi})-\gamma \nabla \times(\nabla \times \vec{\phi})+K \nabla \times \vec{u}-2 K \vec{\phi}=\rho j \frac{\partial^{2} \vec{\phi}}{\partial t^{2}}, \\
K^{*} \nabla^{2} \Phi=\rho c^{*} \frac{\partial}{\partial t}\left(1-a \nabla^{2}\right) \Phi+\nu \Phi_{0}\left(\frac{\partial}{\partial t}\right)(\nabla \cdot \vec{u}),
\end{gathered}
$$

and the constitutive relations are

$$
\begin{aligned}
& t_{i j}=\lambda u_{r, r} \delta_{i j}+\mu\left(u_{i, j}+u_{j, i}\right)+K\left(u_{j, i}-\varepsilon_{i j r} \phi_{r}\right)-\nu T \delta_{i j}, \\
& m_{i j}=\alpha \phi_{r, r} \delta_{i j}+\beta \phi_{i, j}+\gamma \phi_{j, i}, \quad i, j, r=1,2,3
\end{aligned}
$$


where $\nabla^{2}$ is the Laplacian operator, $\lambda$ and $\mu$ are Lame's constants. $K$, $\alpha, \beta$ and $\gamma$ are micropolar constants. $t_{i j}$ are the components of the stress tensor and $m_{i j}$ are the components of couple stress tensor. $\vec{u}$ and $\vec{\phi}$ are the displacement and microrotation vectors, $\rho$ is the density, $\hat{j}$ is the microinertia, $K^{*}$ is the thermal conductivity, $c^{*}$ is the specific heat at constant strain, $T$ is the temperature change, $\nu=(3 \lambda+2 \mu+K) \alpha_{T}$, where $\alpha_{T}$ is the coefficient of linear thermal expansion, $\delta_{i j}$ is the Kronecker delta, $\varepsilon_{i j r}$ is the alternating symbol. Thermodynamic temperature $T$ and conductive temperature $\Phi$ are connected by the relation $T=\left(1-a \nabla^{2}\right) \Phi$.

\section{Formulation of the problem}

We consider two homogeneous, isotropic, micropolar, thermoelastic solid half spaces with two temperature (medium $M_{1}$ ) and (medium $M_{2}$ ) in contact with each other. The rectangular Cartesian co-ordinate system $O x_{1} x_{2} x_{3}$ having origin on the surface $x_{3}=0$ with $x_{3}$-axis pointing vertically into the medium $M_{1}$ is introduced.

For two dimensional problem, we take

$$
\vec{u}=\left(u_{1}\left(x_{1}, x_{3}\right), 0, u_{3}\left(x_{1}, x_{3}\right)\right), \quad \vec{\phi}=\left(0, \phi_{2}\left(x_{1}, x_{3}\right), 0\right)
$$

The following non-dimensional quantities are introduced

$$
\begin{gathered}
x_{1}^{\prime}=\frac{\omega^{*} x_{1}}{c_{1}}, \quad x_{3}^{\prime}=\frac{\omega^{*} x_{3}}{c_{1}}, \quad u_{1}^{\prime}=\frac{\rho \omega^{*} c_{1}}{\nu T_{0}} u_{1}, \quad u_{3}^{\prime}=\frac{\rho \omega^{*} c_{1}}{\nu T_{0}} u_{3}, \\
\phi_{2}^{\prime}=\frac{\rho c_{1}^{2}}{\nu T_{0}} \phi_{2}, \quad t^{\prime}=\omega^{*} t, \quad T^{\prime}=\frac{T}{T_{0}}, \quad \Phi^{\prime}=\frac{\Phi}{\Phi_{0}}, \\
t_{i j}^{\prime}=\frac{1}{\nu T_{0}} t_{i j}, \quad m_{i j}^{\prime}=\frac{\omega^{*}}{c_{1} \nu T_{0}} m_{i j}, \tau_{0}^{\prime}=\omega^{*} \tau_{0}, \quad a^{\prime}=\frac{\omega^{* 2}}{c_{1}^{2}}
\end{gathered}
$$

where $\omega^{*}=\frac{\rho c^{*} c_{1}^{2}}{K^{*}}, \quad c_{1}^{2}=\frac{\lambda+2 \mu+K}{\rho} \quad$ and $T_{0}, \Phi_{0}$ are characteristic temperatures.

The components of displacements $u_{1}$ and $u_{3}$ are related to the potential functions $\phi$ and $\psi$ through the following relation

$$
u_{1}=\frac{\partial \phi}{\partial x_{1}}-\frac{\partial \psi}{\partial x_{3}}, \quad u_{3}=\frac{\partial \phi}{\partial x_{3}}+\frac{\partial \psi}{\partial x_{1}},
$$

Eqs. (6)-(8) with the aid of Eqs. (1)-(3) after suppressing the primes reduce to 


$$
\begin{aligned}
& \nabla^{2} \phi-p_{0}\left(1+\tau_{1} \frac{\partial}{\partial t}\right)\left(1-a \nabla^{2}\right) \Phi-\frac{\partial^{2} \phi}{\partial t^{2}}=0, \\
& \nabla^{2} \psi+a_{1} \phi_{2}-a_{2} \frac{\partial^{2} \psi}{\partial t^{2}}=0, \\
& \nabla^{2} \phi_{2}-a_{3} \nabla^{2} \psi-a_{4} \phi_{2}-a_{5} \frac{\partial^{2} \phi_{2}}{\partial t^{2}}=0, \\
& \nabla^{2} \Phi=a_{6} \frac{\partial}{\partial t}\left(1-a \nabla^{2}\right) \Phi+a_{7}\left(\frac{\partial}{\partial t}\right) \nabla^{2} \phi,
\end{aligned}
$$

where $a_{1}=\frac{K}{\mu+K} \quad, \quad a_{2}=\frac{\rho c_{1}^{2}}{\mu+K}, \quad a_{3}=\frac{K c_{1}^{2}}{\gamma \omega^{* 2}}, \quad a_{4}=2 a_{3}, \quad a_{5}=$ $\frac{\rho \hat{j} c_{1}^{2}}{\gamma}, \quad p_{0}=\frac{\Phi_{0}}{T_{0}} a_{6}=\frac{\rho c^{*} c_{1}^{2}}{K^{*} \omega^{*}}, \quad a_{7}=\frac{\nu^{2} T_{0}}{\rho \mathrm{K}^{*} \omega^{*}}, \quad \nabla^{2}=\frac{\partial}{\partial x_{1}^{2}}+\frac{\partial}{\partial x_{3}^{2}}$

\section{Boundary Conditions}

The boundary conditions at the interface $x_{3}=0$ are given as

$$
\bar{T}_{33}^{\bar{c}}=K_{n}\left[u_{3}-\overline{u_{3}}\right], \quad T_{31}^{-}=K_{t}\left[u_{1}-\overline{u_{1}}\right], \quad \overline{m_{32}}=K_{c}\left[\phi_{2}-\overline{\phi_{2}}\right],
$$

$\overline{K^{*}} \frac{\partial T}{\partial x_{3}}=K_{\theta}[T-\bar{T}], \quad T_{33}=\overline{T_{33}}, \quad T_{31}=\overline{T_{31}}, \quad m_{32}=m_{32}^{-}, \quad K^{*} \frac{\partial T}{\partial x_{3}}=$ $\bar{K}^{*} \frac{\partial \bar{T}}{\partial x_{3}}(13)$

where $K_{n}, K_{t}, K_{c}$ and $K_{\theta}$ are the normal force stiffness, transverse force stiffness, transverse couple stiffness and thermal conductness coefficients of unit layer thickness having dimensions $\frac{N}{m^{3}}, \frac{N}{m^{3}}, \frac{N}{m}$ and $\frac{N}{m \sec K}$.

\section{$5 \quad$ Reflection and Transmission}

We consider Longitudinal displacement wave (LD-wave), Thermal wave ( $\mathrm{T}$ wave), Coupled transverse and microrotational waves (CD-I wave and CD-II wave) propagating through the medium $M_{1}$ which we designate as the region $x_{3}>0$ and incident at the plane $x_{3}=0$ with its direction of propagation making an angle $\theta_{0}$ normal to the surface. Corresponding to each incident wave, we get reflected LD-wave, T-wave, CD-I and CD-II waves in medium $M_{1}$ and transmitted LD-wave, T-wave, CD-I and CD-II waves in medium $M_{2}$. We write all the variables without bar in medium $M_{1}$ and attach bar to denote the variables in medium $M_{2}$ as shown in Fig.1. form

In order to solve the equations (15)-(20), we assume the solutions of the 
$\left\{\phi, \Phi, \psi, \phi_{2}\right\}=\left\{\tilde{\phi}, \tilde{\Phi}, \tilde{\psi}, \tilde{\phi_{2}}\right\} e^{\iota\left\{k\left(x_{1} \sin \theta-x_{3} \cos \theta\right)-\omega t\right\}}$

where $k$ is the wave number and $\omega$ is the angular frequency and $\tilde{\phi}, \tilde{\Phi}, \widetilde{\psi}, \widetilde{\phi_{2}}$ are arbitrary constants.

Making use of equation (14) in equations (9)-(12), we obtain

$$
\begin{aligned}
& V^{4}+D_{1} V^{2}+E_{1}=0, \\
& V^{4}+D_{2} V^{2}+E_{2}=0,
\end{aligned}
$$

where

$$
\begin{aligned}
& D_{1}=-\left(\frac{a_{1} a_{3}}{\omega^{2} a_{2}}+1\right) \frac{1}{\left(a_{5}-\frac{a_{4}}{\omega^{2}}\right)}-\frac{1}{a_{2}}, \quad E_{1}=\frac{1}{\left(a_{5}-\frac{a_{4}}{\omega^{2}}\right) a_{2}}, \\
& D_{2}=\frac{-1+\left(a-\frac{1}{\omega^{2}}\right) i a_{6} \omega-a_{7} i \omega p_{0}}{a_{6} \frac{i}{\omega}}, \quad E_{2}=\frac{1-a \omega^{2}\left[-i a_{7} p_{0} \frac{1}{\omega^{2}}-a_{6} \frac{i}{\omega}\right]}{a_{6} \frac{i}{\omega}}
\end{aligned}
$$

and $V^{2}=\frac{\omega^{2}}{k^{2}}$

Equation (15) and (16) are quadratic in $V^{2}$, therefore the roots of these equations give four values of $V^{2}$. Corresponding to each value of $V^{2}$ in equation (15), there exist two types of waves in solid medium in decreasing order of their velocities, namely a LD-wave, T-wave. Similarly corresponding to each value of $V^{2}$ in equation (16), there exist two types of waves in solid medium, namely a CD-I wave, CD-II wave. Let $V_{1}, V_{2}$ be the velocities of reflected LD-wave, T-wave and $V_{3}, V_{4}$ be the velocities of reflected CD-I wave, CD-II wave in medium $M_{1}$.

In view of equation (14), the appropriate solutions of equations (9)-(12) for medium $M_{1}$ and medium $M_{2}$ are assumed in the form

Medium $M_{1}$ :

$$
\begin{aligned}
& \{\phi, \Phi\}=\sum_{i=1}^{2}\left\{1, f_{i}\right\}\left[S_{0 i} e^{\ell\left\{k_{i}\left(x_{1} \sin \theta_{0 i}-x_{3} \cos \theta_{0 i}\right)-\omega_{i} t\right\}}+P_{i}\right], \\
& \left\{\psi, \phi_{2}\right\}=\sum_{j=3}^{4}\left\{1, f_{j}\right\}\left[T_{0 j} e^{\ell\left\{k_{j}\left(x_{1} \sin \theta_{0 j}-x_{3} \cos \theta_{0 j}\right)-\omega_{j} t\right\}}+P_{j}\right],
\end{aligned}
$$

where

$$
f_{i}=\frac{\iota \omega\left(1-\frac{1}{V_{i}^{2}}\right)}{p_{0}\left(1+\frac{a \omega^{2}}{V_{i}^{2}}\right) \frac{\iota}{\omega}}, \quad f_{j}=\frac{-\omega^{2}\left(a_{2}-\frac{1}{V_{j}^{2}}\right)}{a_{1}}
$$

and $P_{i}=S_{i} e^{\ell\left\{k_{i}\left(x_{1} \sin \theta_{0 i}+x_{3} \cos \theta_{0 i}\right)-\omega_{i} t\right\}}, P_{j}=T_{j} e^{\ell\left\{k_{j}\left(x_{1} \sin \theta_{0 j}+x_{3} \cos \theta_{0 j}\right)-\omega_{j} t\right\}}$ 
Medium $M_{2}$ :

$$
\begin{aligned}
& \{\bar{\phi}, \bar{\Phi}\}=\sum_{i=1}^{2}\left\{1, \overline{f_{i}}\right\}\left[\overline{S_{0 i}} e^{\iota\left\{\overline{k_{i}}\left(x_{1} \sin \overline{\theta_{i}}-x_{3} \cos \overline{\theta_{i}}\right)-\overline{\omega_{i}} t\right\}}\right], \\
& \left\{\bar{\psi}, \overline{\phi_{2}}\right\}=\sum_{j=3}^{4}\left\{1, \overline{f_{j}}\right\}\left[\overline{T_{0 j}} e^{\iota\left\{\overline{k j}\left(x_{1} \sin \overline{\theta_{j}}-x_{3} \cos \overline{\theta_{j}}\right)-\overline{\omega_{j}} t\right\}}\right],
\end{aligned}
$$

and $S_{0 i}, T_{0 j}$ are the amplitudes of incident (LD-wave, T-wave) and (CD-I, CD-II) waves respectively. $S_{i}$ and $T_{j}$ are the amplitudes of reflected (LD-wave, T-wave) and (CD-I, CD-II) waves and $\underset{i}{\bar{S}}, \underset{j}{\bar{T}}$ are the amplitudes of transmitted (LD-wave, T-wave) and (CD-I, CD-II) waves respectively.

We use the following extension of the Snell's law in order to satisfy the boundary conditions

$$
\frac{\sin \theta_{0}}{V_{0}}=\frac{\sin \theta_{1}}{V_{1}}=\frac{\sin \theta_{2}}{V_{2}}=\frac{\sin \theta_{3}}{V_{3}}=\frac{\sin \theta_{4}}{V_{4}}=\frac{\sin \overline{\theta_{1}}}{\overline{V_{1}}}=\frac{\sin \overline{\theta_{2}}}{\overline{V_{2}}}=\frac{\sin \overline{\theta_{3}}}{\overline{V_{3}}}=\frac{\sin \overline{\theta_{4}}}{\overline{V_{4}}}
$$

where

$$
V_{j}=\frac{\omega}{k_{j}} \overline{V_{j}}=\frac{\omega}{\overline{k_{j}}}(j=1,2,3,4) a t x_{3}=0
$$

Making use of $\phi, \psi, \Phi$ and $\phi_{2}$ in boundary conditions (13), equations (4)(8) and equations (21) and (22), we obtain a system of eight non-homogeneous equations in the following form

$$
\sum_{j=1}^{8} a_{i j} Z_{j}=Y_{i} ; \quad(i=1,2,3,4,5,6,7,8)
$$

where

$$
\begin{aligned}
& a_{1 i}=\left(d_{1}+d_{2} B_{i}\right) \frac{\omega^{2}}{V_{i}^{2}}+p_{0}\left(1+a \frac{\omega^{2}}{V_{i}^{2}}\right) f_{i}, a_{1 j}=d_{2} \frac{\omega^{2}}{V_{\mathrm{j}} V_{0}} \sin \theta_{0} \sqrt{B_{j}}, a_{1 k}= \\
& -\left[\left(\overline{d_{1}}+\overline{d_{2}}\left(R_{i}\right)\right) \frac{\omega^{2}}{\overline{V_{i}^{2}}}+\left(1+\bar{a} \frac{\omega^{2}}{\bar{V}_{i}^{2}}\right) \overline{f_{i}}\right], a_{1 l}=-\bar{d}_{2} \frac{\omega^{2}}{\overline{V_{j}} \mathrm{~V}_{0}} \sin \theta_{0} \sqrt{R_{j}}, a_{2 i}= \\
& -\left(2 d_{4}+d_{5}\right) \frac{\omega^{2}}{V_{i} V_{0}} \sin \theta_{0} \sqrt{B_{i}}, a_{4 l}=\overline{d_{2}} \frac{\omega^{2}}{\overline{V_{j} V_{0}}} \sqrt{R_{j}}-\iota K_{n} \frac{\omega}{V_{0}} \sin \theta_{0}, \\
& a_{2 j}=2 d_{4} \frac{\omega^{2}}{V_{j}^{2}} B_{j}-d_{5} \frac{\omega^{2}}{V_{0}^{2}} \sin ^{2} \theta_{0}-d_{5} f_{j}, a_{2 k}=-\left(2 \overline{d_{4}}+\overline{d_{5}}\right) \frac{\omega^{2}}{\overline{V_{i} V_{0}}} \sin \theta_{0} \sqrt{R_{i}}, \\
& a_{2 l}=-\left[2 \overline{d_{4}} \frac{\omega^{2}}{\overline{V_{j}^{2}}}\left(1-2 \frac{\overline{V_{j}^{2}}}{V_{0}^{2}} \sin ^{2} \theta_{0}\right)-\overline{d_{5}}\left(\frac{\omega^{2}}{V_{0}^{2}} \sin ^{2} \theta_{0}+\overline{\left.f_{j}\right)}\right],\right.
\end{aligned}
$$




$$
\begin{aligned}
& a_{3 i}=0, \quad a_{3 j}=\iota \frac{V_{j}}{V_{0}} \sin \theta_{0} f_{j}, \quad a_{3 k}=0, \quad a_{3 l}=-\iota \frac{\omega}{\overline{V_{j}}} \sqrt{R_{j} f_{j}}, \\
& a_{4 i}=\iota \frac{\omega}{\bar{V}_{i}} K_{n} \sqrt{B_{i}}, a_{4 j}=\iota K_{n} \frac{\omega}{V_{0}} \sin \theta_{0}, \quad a_{4 k}=\iota K_{n} \frac{\omega}{\overline{V_{i}}} \sqrt{R_{i}}+\frac{\omega^{2}}{\overline{V_{i}^{2}}}\left(\overline{d_{1}}+\right. \\
& \left.\overline{d_{2}} R_{i}\right)+\left(1+\bar{a} \frac{\overline{V_{i}^{2}}}{\omega^{2}}\right) \overline{f_{i}} \text {, } \\
& a_{5 i}=\iota K_{t} \frac{\omega}{\mathrm{V}_{0}} \sin \theta_{0}, a_{5 j}=-\iota K_{t} \frac{\omega}{V_{j}} \sqrt{B_{j}} \\
& a_{5 k}=\left(2 \overline{d_{4}}+\overline{d_{5}}\right) \frac{\omega^{2}}{\overline{V_{i}} V_{0}} \sin \theta_{0} \sqrt{R_{i}}-\iota K_{t} \frac{\omega}{V_{0}} \sin \theta_{0}, \\
& a_{5 l}=\frac{\omega^{2}}{\overline{V_{\mathrm{j}}^{2}}} \overline{d_{4}}\left(1-2 \frac{\overline{V_{j}^{2}}}{V_{0}^{2}} \sin ^{2} \theta_{0}\right)-\left(\frac{\omega^{2}}{V_{0}^{2}} \sin ^{2} \theta_{0}+\overline{f_{j}}\right) \overline{d_{5}}+\iota K_{t} \frac{\omega}{\overline{V_{j}}} \sqrt{R_{j}}, \\
& a_{6 i}=0, \quad a_{6 j}=K_{c} f_{j}, \quad a_{6 k}=0, \quad a_{6 l}=\iota p_{1} \frac{\omega}{\overline{V_{j}}} \overline{f_{j}} \sqrt{R_{j}}-K_{c} \overline{f_{j}}, \\
& a_{7 i}=\left(1+a \frac{\omega^{2}}{V_{i}^{2}}\right) f_{i} K_{\theta}, \quad a_{7 j}=0, \quad a_{7 k}=\left(1+\bar{a} \frac{\omega^{2}}{\overline{V_{i}^{2}}}\right)\left(\iota p_{2} \frac{\omega}{\overline{V_{i}}} \overline{f_{i}} \sqrt{R_{i}}-\overline{f_{i}} K_{\theta}\right), \\
& a_{7 l}=0, a_{8 i}=\left(1+a \frac{\omega^{2}}{V_{i}^{2}}\right) f_{i}, a_{8 j}=\left(1+a \frac{\omega^{2}}{V_{i}^{2}}\right) f_{j}, a_{8 k}=-\left(1+\bar{a} \frac{\omega^{2}}{\overline{V_{i}^{2}}}\right) \overline{f_{i}}, \\
& a_{8 l}=0 \\
& d_{1}=\frac{\lambda}{\rho c_{1}^{2}}, \quad d_{2}=\frac{(2 \mu+\kappa)}{\rho c_{1}^{2}}, \quad d_{4}=\frac{2 \mu}{\rho c_{1}^{2}}, \quad d_{5}=\frac{d_{2}}{2}, \quad p_{1}=\frac{\bar{\gamma} \omega^{*}}{\gamma}, \\
& p_{2}=\frac{K_{1}^{*}}{K^{*}}, \quad B_{i}=\left(1-\frac{V_{i}^{2}}{V_{0}^{2}} \sin ^{2} \theta_{0}\right), B_{j}=\left(1-\frac{V_{j}^{2}}{V_{0}^{2}} \sin ^{2} \theta_{0}\right), R_{i}=\left(1-\frac{\overline{V_{i}^{2}}}{V_{0}^{2}} \sin ^{2} \theta_{0}\right) \text {, } \\
& R_{j}=\left(1-\frac{\overline{V_{j}^{2}}}{V_{0}^{2}} \sin ^{2} \theta_{0}\right) \\
& (i=1,2, j=3,4, k=5,6 \text {, and } l=7,8) \\
& \text { and } \\
& Z_{1}=\frac{S_{1}}{A^{*}}, Z_{2}=\frac{S_{2}}{A^{*}}, Z_{3}=\frac{T_{3}}{A^{*}}, Z_{4}=\frac{T_{4}}{A^{*}}, \\
& Z_{5}=\frac{\overline{S_{1}}}{\overline{A^{*}}}, Z_{6}=\frac{\overline{S_{2}}}{\overline{A^{*}}}, \quad Z_{7}=\frac{\overline{T_{3}}}{A^{*}}, Z_{8}=\frac{\overline{T_{4}}}{A^{*}}
\end{aligned}
$$

such that $Z_{1}, Z_{2}, Z_{3}, Z_{4}$ are the complex amplitude ratios of reflected LDwave, T-wave and coupled CD-I, CD-II waves in medium $M_{1}$ and $Z_{5}, Z_{6}, Z_{7}$, $Z_{8}$ are the complex amplitude ratios of transmitted LD-wave, T-wave and coupled CD-I, CD-II waves in medium $M_{2}$.

(1) For incident LD-wave: 


$$
\begin{gathered}
A^{*}=S_{01}, \quad S_{02}=T_{03}=T_{04}=0, \quad Y_{1}=-a_{11}, \\
Y_{2}=a_{21}, \quad Y_{3}=a_{31}=0, \quad Y_{4}=a_{41}, \\
Y_{5}=a_{51}, \quad Y_{6}=-a_{61}, \quad Y_{7}=a_{71}=0, \quad Y_{8}=-a_{81}
\end{gathered}
$$

(2) For incident T-wave:

$$
\begin{gathered}
A^{*}=S_{02}, \quad S_{01}=T_{03}=T_{04}=0, \quad Y_{1}=-a_{12}, \\
Y_{2}=a_{22}, \quad Y_{3}=a_{32}=0, \quad Y_{4}=a_{42}, \\
Y_{5}=a_{52}, \quad Y_{6}=-a_{62}, \quad Y_{7}=a_{72}=0, \quad Y_{8}=-a_{82}
\end{gathered}
$$

(3) For incident CD-I wave:

$$
\begin{gathered}
A^{*}=T_{03}, \quad S_{01}=S_{02}=T_{04}=0, \quad Y_{1}=a_{13}, \\
Y_{2}=-a_{23}, \quad Y_{3}=a_{33}, \quad Y_{4}=a_{43}, \\
Y_{5}=-a_{53}, \quad Y_{6}=a_{63}=0, \quad Y_{7}=-a_{73}, \quad Y_{8}=a_{83}=0
\end{gathered}
$$

(4) For incident CD-II wave:

$$
\begin{gathered}
A^{*}=T_{04}, \quad S_{01}=S_{02}=T_{03}=0, \quad Y_{1}=a_{14}, \\
Y_{2}=-a_{24}, \quad Y_{3}=a_{34}, \quad Y_{4}=a_{44}, \\
Y_{5}=-a_{54}, \quad Y_{6}=a_{64}=0, \quad Y_{7}=-a_{74}, \quad Y_{8}=a_{84}=0
\end{gathered}
$$

\section{Particular cases}

\section{Case I: Normal force stiffness}

If $K_{t} \rightarrow \infty, K_{c} \rightarrow \infty, K_{n} \neq 0, K_{\theta} \rightarrow \infty$ then eq.(23) yield the corresponding expression for the normal force stiffness with the changed values of $a_{i j}$ as

$$
a_{5 i}=\iota \frac{\omega}{V_{0}} \sin \theta_{0}, a_{5 j}=-\iota \frac{\omega}{V_{j}} \sqrt{B_{j}}, a_{5 k}=-\iota \frac{\omega}{V_{0}} \sin \theta_{0}, a_{5 l}=\iota \frac{\omega}{\overline{V_{j}}} \sqrt{R_{j}},
$$

$a_{7 l}=0, a_{6 i}=0, a_{6 j}=f_{j}, a_{6 k}=0, a_{6 l}=-\overline{f_{j}}, a_{7 i}=\left(1+a \frac{\omega^{2}}{V_{i}^{2}}\right) f_{i}, a_{7 j}=$ $0, \quad a_{7 k}=-\left(1+\bar{a} \frac{\omega^{2}}{\overline{V_{i}^{2}}}\right) \overline{f_{i}}, i=1,2, j=3,4, k=5,6$, and $\left.l=7,8\right)$

\section{Case II: Transverse force stiffness}

As $K_{t} \neq 0, K_{c} \rightarrow \infty, K_{n} \rightarrow \infty, K_{\theta} \rightarrow \infty$, we obtain a system of eight non-homogeneous equations as given by equation (23) for the transverse force stiffness with the changed values of $a_{i j}$ as $a_{4 i}=\iota \frac{\omega}{V_{i}} \sqrt{1-\frac{V_{i}^{2}}{V_{0}^{2}} \sin ^{2} \theta_{0}}$, $a_{4 j}=\iota \frac{\omega}{V_{0}} \sin \theta_{0}, \quad a_{4 k}=\iota \frac{\omega}{V_{i}} \sqrt{R_{i}}, \quad a_{4 l}=-\iota \frac{\omega}{V_{0}} \sin \theta_{0}$, 
$a_{6 i}=0, \quad a_{6 j}=f_{j}, \quad a_{6 k}=0, \quad a_{6 l}=-\overline{f_{j}}, a_{7 i}=\left(1+a \frac{\omega^{2}}{V_{i}^{2}}\right) f_{i}, a_{7 j}=$

$0, a_{7 k}=-\left(1+\bar{a} \frac{\omega^{2}}{\overline{V_{i}^{2}}}\right) \overline{f_{i}}, a_{77}=a_{78}=0$,

$(i=1,2, j=3,4, k=5,6$, and $l=7,8)$

Case III:Transverse couple stiffness

As $K_{t} \rightarrow \infty, K_{c} \neq 0, K_{n} \rightarrow \infty, K_{\theta} \rightarrow \infty$, the boundary conditions reduce to the transverse couple stiffness, yielding a system of eight nonhomogeneous equations as given by equation (23) with the changed values of $a_{i j}$ as

$a_{4 i}=\iota \frac{\omega}{\mathrm{V}_{i}} \sqrt{B_{i}}, a_{4 j}=\iota \frac{\omega}{\mathrm{V}_{0}} \sin \theta_{0}, \quad a_{4 k}=\iota \frac{\omega}{V_{i}} \sqrt{R_{i}}, \quad a_{4 l}=-\iota \frac{\omega}{V_{0}} \sin \theta_{0}$, $a_{5 i}=\iota \frac{\omega}{V_{0}} \sin \theta_{0} \quad, \quad a_{5 j}=-\iota \frac{\omega}{V_{j}} \sqrt{B_{j}}, \quad a_{5 k}=-\iota \frac{\omega}{V_{0}} \sin \theta_{0}, a_{5 l}=$ $\iota \frac{\omega}{\bar{V}_{j}} \sqrt{R_{j}}, \quad a_{7 i}=\left(1+a \frac{\omega^{2}}{V_{i}^{2}}\right) f_{i}, \quad a_{7 j}=0, \quad a_{7 k}=-\left(1+\bar{a} \frac{\omega^{2}}{{\overline{V_{i}^{2}}}^{2}}\right) \overline{f_{i}}, a_{7 l}=0$,

$(i=1,2, j=3,4, k=5,6$ and $l=7,8)$

Case IV: Thermal conductness

If $K_{t} \rightarrow \infty, K_{c} \rightarrow \infty, K_{n} \rightarrow \infty, K_{\theta} \neq 0$ correspond, then the corresponding results for the case of thermal conductness and we obtain a system of eight non-homogeneous equations as given by equation (23) with the changed values of $a_{i j}$ as

$$
\begin{aligned}
& a_{4 i}=\iota \frac{\omega}{\mathrm{V}_{i}} \sqrt{B_{i}}, a_{4 j}=\iota \frac{\omega}{\mathrm{V}_{0}} \sin \theta_{0}, \quad a_{4 k}=\iota \frac{\omega}{V_{i}} \sqrt{R_{i}}, \\
& a_{4 l}=-\iota \frac{\omega}{V_{0}} \sin \theta_{0}, \quad a_{5 i}=\iota \frac{\omega}{\mathrm{V}_{0}} \sin \theta_{0}, \quad a_{5 j}=-\iota \frac{\omega}{V_{j}} \sqrt{B_{j}}, \quad a_{5 k}= \\
& -\iota \frac{\omega}{V_{0}} \sin \theta_{0}, a_{5 l}=\iota \frac{\omega}{V_{j}} \sqrt{R_{j}}, a_{6 i}=0, \quad a_{6 j}=f_{j}, \quad a_{6 k}=0, \quad a_{6 l}= \\
& -\overline{f_{j}}, \\
& \quad(i=1,2, j=3,4, k=5,6, \text { and } l=7,8) \\
&
\end{aligned}
$$

Case V: Perfect bonding

By putting the values $K_{t} \rightarrow \infty, K_{c} \rightarrow \infty, K_{n} \rightarrow \infty, K_{\theta} \rightarrow \infty$ in equation (23), we obtain a system of eight non-homogeneous equations as given by equation (23) with the changed values of $a_{i j}$ as

$$
\begin{gathered}
a_{4 i}=\iota \frac{\omega}{\mathrm{V}_{i}} \sqrt{B_{i}}, a_{4 j}=\iota \frac{\omega}{\mathrm{V}_{0}} \sin \theta_{0}, \quad a_{4 k}=\iota \frac{\omega}{\bar{V}_{i}} \sqrt{R_{i}}, \\
a_{4 l}=-\iota \frac{\omega}{V_{0}} \sin \theta_{0}, \quad a_{5 i}=\iota \frac{\omega}{\mathrm{V}_{0}} \sin \theta_{0}, \quad a_{5 j}=-\iota \frac{\omega}{V_{j}} \sqrt{B_{j}}, \quad a_{5 k}= \\
-\iota \frac{\omega}{V_{0}} \sin \theta_{0}, a_{5 l}=\iota \frac{\omega}{\overline{V_{j}}} \sqrt{R_{j}}, a_{6 i}=0, \quad a_{6 j}=f_{j}, \quad a_{6 k}=0, \quad a_{6 l}= \\
-\overline{f_{j}}, \quad a_{7 i}=\left(1+a \frac{\omega^{2}}{V_{i}^{2}}\right) f_{i}, \quad a_{7 j}=0, \quad a_{7 k}=-\left(1+\bar{a} \frac{\omega^{2}}{\overline{V_{i}^{2}}}\right) \overline{f_{i}}, a_{7 l}=0, \\
(i=1,2, j=3,4, k=5,6, \text { and } l=7,8)
\end{gathered}
$$

\section{Special Case}

If two temperature parameters vanish i.e. $a=0, \bar{a}=0$ with $\Phi_{0}=\overline{\Phi_{0}}$ and $\overline{\Phi_{0}}=T_{0}$ yield the amplitude ratios at the imperfect boundary of two micropolar thermoelastic solid half spaces with the changed values of $a_{i j}$ as 


$$
\begin{gathered}
a_{1 k}=-\left[\left(\overline{d_{1}}+\overline{d_{2}} R_{i}\right) \overline{\frac{V_{i}^{2}}{\omega^{2}}}+\overline{f_{i}}\right], a_{4 k}=\iota K_{n} \frac{\omega}{\bar{V}_{i}} \sqrt{R_{i}}+\frac{\omega^{2}}{\overline{V_{i}^{2}}}\left(\overline{d_{1}}+\overline{d_{2}} R_{i}\right)+\overline{f_{i}}, \\
a_{6 j}=K_{c} f_{j}, a_{6 l}=\iota p_{1} \frac{\omega}{\overline{V_{j}}} \overline{f_{j}} \sqrt{\overline{R_{j}}}-K_{c} \overline{f_{j}}, a_{7 i}=f_{i} K_{\theta}, a_{7 k}=\left(\iota p_{2} \frac{\omega}{\overline{V_{i}}} \overline{f_{i}} \sqrt{R_{i}}-\right. \\
\left.\overline{f_{i}} K_{\theta}\right), a_{8 i}=f_{i}, a_{8 j}=f_{j}, a_{8 k}=-\overline{f_{i}},(i=1,2, j=3,4, k=5,6, \text { and } l=7,8)
\end{gathered}
$$

\section{Numerical results and discussion}

The following values of relevant parameters for both the half spaces for numerical computations are taken.

Following Eringen[39 ], the values of micropolar constants for medium $M_{1}$ are taken :

$\lambda=9.4 \times 10^{10} \mathrm{Nm}^{-2}, \mu=4.0 \times 10^{10} \mathrm{Nm}^{-2}, \kappa=1.0 \times 10^{10} \mathrm{Nm}^{-2}, \gamma=$ $7.79 \times 10^{-10} \mathrm{~N}, \hat{j}=0.002 \times 10^{-17} \mathrm{~m}^{2}, \rho=1.74 \times 10^{3} \mathrm{Kgm}^{-3}$,

and thermal parameters are taken from Dhaliwal and Singh [40]:

$\nu=0.268 \times 10^{5} \mathrm{Nm}^{-2} K^{-1}, \quad c^{*}=0.104 \times 10^{4} N m K g^{-1} K^{-1}, \quad a=0.5 m^{2}$,

$$
\begin{gathered}
T_{0}=0.298 K, \quad \Phi_{0}=0.292 K, \quad K^{*}=1.7 \times 10^{2} \mathrm{Nsec}^{-1} K^{-1}, \\
\tau_{0}=8.13 \times 10^{-15} \mathrm{sec}, \quad \omega=1
\end{gathered}
$$

Following Gauthier [41], the values of micropolar constants for medium $M_{2}$ are taken as:

$$
\begin{gathered}
\bar{\lambda}=7.59 \times 10^{10} \mathrm{Nsecm}^{-2}, \quad \bar{\mu}=0.00189 \times 10^{13} \mathrm{Nsecm}^{-2}, \\
\bar{\kappa}=0.0149 \times 10^{9} \mathrm{Nsecm}^{-2}, \quad \bar{\gamma}=0.0000268 \mathrm{Nsec}, \bar{\rho}=2.19 \times 10^{3} \mathrm{Kgm}^{-3},
\end{gathered}
$$

Thermal parameters for the medium $M_{2}$ are taken as:

$$
\begin{aligned}
& \quad \overline{T_{0}}=0.296 K, \quad \overline{\Phi_{0}}=0.295 K, \quad \overline{K^{*}}=2.04 \times 10^{2} \mathrm{Nsec}^{-1} K^{-1}, \\
& \bar{\nu}=0.2603 \times 10^{7} \mathrm{Nm}^{-2} K^{-1}, \quad \overline{c^{*}}=0.0921 \times 10^{4} \mathrm{JKg}^{-1} \mathrm{~K}^{-1}, \quad \bar{a}=0.1 \mathrm{~m}^{2} \\
& \overline{\tau_{0}}=7.13 \times 10^{-15} \mathrm{sec}
\end{aligned}
$$

The values of amplitude ratios have been computed at different angles of incidence. 
In Figs. 2-25, for CT-theory, we represent the solid line for incident wave for stiffness (GT), small dashes line for incident wave for transverse couple stiffness (KC), medium dashes line for incident wave for normal force stiffness $(\mathrm{KN})$, solid line with solid circles for incident wave for thermal conductness (KQ), solid line with plus sign for incident wave for transverse force stiffness (KT), solid line with crosses for incident wave for thermoelastic solid (TS) and solid line with triangles for incident wave for thermoelastic solid with two temperature without stiffness (WS).

\subsection{Incident LD-Wave}

Variations of amplitude ratios $\left|Z_{i}\right| ; 1 \leq i \leq 8$ with the angle of incidence $\theta_{0}$, for incident LD-wave are shown in Figs. 2 through 9.

Fig. 2 depicts that the values of $\left|Z_{1}\right|$ for all the stiffnesses increase in the whole range, except the values of GT which oscillate in the whole range of $\theta_{0}$. Also the values for WS remain more than the values for all the other stiffnesses in the whole range.It is evident from fig. 3 that the values of $\left|Z_{2}\right|$ for $\mathrm{KN}$ remain less than the values for all the other stiffnesses. The maximum value is attained for WS near $\theta_{0}=90^{\circ}$.

Fig. 4 shows that the values for $\left|Z_{3}\right|$ for all the stiffnesses increase for all the values of $\theta$ except the values for GT which decrease near the grazing incidence. The values for $\mathrm{KC}$ remain more than the values for all the other stiffnesses in the whole range. The values for TS remain less than the values for all the other stiffnesses for all the values of $\theta$. Fig. 5 depicts that the behavior of variation of $\left|Z_{4}\right|$ is similar to that of $\left|Z_{3}\right|$ with difference in their magnitude values.

From fig. 6, it is evident that the values of $\left|Z_{5}\right|$ for GT remain less than the values for all the other stiffnesses. The values for all the stiffnesses increase while the values for GT decrease in the whole range. The maximum value is attained by WS near the grazing incidence. Fig. 7 shows that the values of $\left|Z_{6}\right|$ for KT remain greater than all the other stiffnesses in the whole range except near the grazing incidence that reveals the effect of transverse force stiffness. It is noticed that the behavior of variation of $\left|Z_{6}\right|$ is similar to that of $\left|Z_{5}\right|$.

It is evident from figs. 8 that the values of $\left|Z_{7}\right|$ for all the stiffnesses increase from normal incidence to grazing incidence, while the values for WS oscillate in the whole range. The values for GT are more than the values for all the other stiffnesess for all the values of $\theta$. There is slight difference in the magnitude of GT and KN in the whole range of $\theta_{0}$. It is noticed from fig. 9 that there is only slight difference in the amplitude of $\left|Z_{7}\right|$ and $\left|Z_{8}\right|$. 


\subsection{Incident $\mathrm{T}$}

\section{-Wave}

Variations of amplitude ratios $\left|Z_{i}\right| ; 1 \leq i \leq 8$, with the angle of incidence $\theta_{0}$, for incident T-wave are shown in Figs. 10 through 17.

Fig. 10 depicts that the values of $\left|Z_{1}\right|$ for $\mathrm{KQ}$ remain more than the values for $\mathrm{KN}, \mathrm{KT}, \mathrm{GT}, \mathrm{KC}$ and $\mathrm{WS}$ in the whole range that shows the effect of thermal conductness. Also it is noticed that the values for GT remain less than the values for all the other stiffnesses.

Fig. 11 shows that the values of $\left|Z_{2}\right|$ for GT oscillate with increase in $\theta_{0}$ and attains peak value in the range $25^{0}<\theta_{0}<35^{0}$ and remain more than the values for all the other stiffnesses in the whole range, except near the grazing incidence. The values for WS remain greater than the values for $\mathrm{KC}, \mathrm{KQ}, \mathrm{KN}$, $\mathrm{KT}$ and TS in the whole range.

It can be noticed from fig. 12 that the values of $\left|Z_{3}\right|$ for all the stiffnesses increase in the whole range, while the values for GT oscillate and attain maximum value in the range $25^{0}<\theta_{0}<35^{0}$. The maximum value is attained by TS near the grazing incidence. Fig. 13 depicts that the behavior of variation of $\left|Z_{4}\right|$ for $\mathrm{KT}$ and $\mathrm{KQ}$ is similar with slight difference in their magnitude. The values for TS remain more than the values for all the other stiffnesses in the whole range that reveal the effect of two temperature.

Fig. 14 shows the values of $\left|Z_{5}\right|$ for WS remain more than the values for all the other stiffnesses for all the values of $\theta$. It is noticed that the values for all the stiffnesses increase, while the values for $\mathrm{GT}$ and $\mathrm{KC}$ oscillate with increase in $\theta_{0}$, due to the effect of stiffness. Fig. 15 shows the values of $\left|Z_{6}\right|$ for GT attains maximum value near the grazing incidence. The values for $\mathrm{KN}$ remain more than the values for KC, KT, KQ, TS and WS in the whole domain. The values for GT attain peak value in the range $30^{\circ}<\theta_{0}<35^{0}$ due to the effect of stiffness.

Fig. 16 shows that the values of $\left|Z_{7}\right|$ for $\mathrm{GT}$ are more than the values for all the other stiffnesses, The values for GT and KN oscillate, while all the other stiffnesses show increase in value. Fig. 17 shows that the behavior of variation of $\left|Z_{8}\right|$ is similar to that of $\left|Z_{7}\right|$ with difference in magnitude.

\subsection{Incident CD-I Wave}

Variations of amplitude ratios $\left|Z_{i}\right| ; 1 \leq i \leq 8$, with the angle of incidence $\theta_{0}$ , for incident CD-I wave are shown in Figs. 18 through 25.

Fig. 18 depicts that the values of $\left|Z_{1}\right|$ for GT increase in the whole range and then decrease sharply near the grazing incidence. Also it is noticed that the values for WS decrease very sharply at the normal incidence. Also the values for TS attain maximum value in the range $40^{\circ}<\theta_{0}<60^{\circ}$. It is depicted 
from fig. 19 that the behavior of variation of $\left|Z_{2}\right|$ is similar as that for $\left|Z_{1}\right|$ with difference in magnitude. The values for all the stiffnesses oscillate in the whole range. The maximum value is attained by GT near the grazing incidence.

It is noticed from fig. 20 that the values of $\left|Z_{3}\right|$ for $\mathrm{KQ}$ and $\mathrm{KN}$ decrease from normal incidence to grazing incidence. Also the values for $\mathrm{KC}$ remain greater than the values for all the stiffnesses in the range $0^{0}<\theta_{0}<82^{0}$. The values for WS are smaller than the values for all the other stiffnesses in the whole range of $\theta_{0}$.

Fig. 21 depicts that the values of $\left|Z_{4}\right|$ for all the stiffnesses decrease in the whole range, except the values for GT and TS which oscillate in the whole range and remain less than the values for all the other stiffnesses. The maximum value is attained by $\mathrm{KC}$ at the normal incidence. Fig. 22 shows that the values of $\left|Z_{5}\right|$ for WS decrease sharply and the value for GT increase for all the values of $\theta$ and then decrease sharply near the grazing incidence. The behavior of variation of $\mathrm{KQ}$ and $\mathrm{KT}$ is similar with slight difference in magnitude values.

Fig. 23 depicts that the values of $\left|Z_{6}\right|$ for all the stiffnesses oscillate in the whole range. The maximum value is attained by WS near the normal incidence. It is noticed from fig. 24 that the values of $\left|Z_{7}\right|$ for TS decrease from normal incidence to grazing incidence and remain more than the values for all the other stiffnesses in the whole range. It is noticed from fig. 25 that behavior of variation of $\left|Z_{8}\right|$ is similar as that of $\left|Z_{7}\right|$ with difference in their magnitude values.

\section{Conclusion}

In the present paper, the expressions for reflection and transmission coefficients of various reflected and transmitted waves has been derived for the normal force stiffness, transverse force stiffness, transverse couple stiffness, thermal conductness and perfect bonding. It is observed that when LD-wave is incident, the values of amplitude ratios for all the stiffnesses increase, while the values for GT oscillate in the whole range. It is evident that the maximum value is attained by WS near the grazing incidence for $\left|Z_{i}\right| ; 1 \leq i \leq 6$ . Also when T-wave is incident, the values of amplitude ratios for TS attain peak value in the intermediate range due to the effect of stiffness. The values of amplitude ratios for all the boundary stiffnesses follow oscillatory pattern (when CD-I wave is incident). It is also observed that the values of amplitude ratios $\left|Z_{1}\right|,\left|Z_{2}\right|,\left|Z_{5}\right|$ and $\left|Z_{6}\right|$ for WS decrease very sharply near the

normal incidence that reveals the effect of perfect bonding. The problem is of geographical interest and the results are supposed to be useful in theoreti- 
cal and observational studies of wave propagation in more realistic models of micropolar solids present in the earths interior.

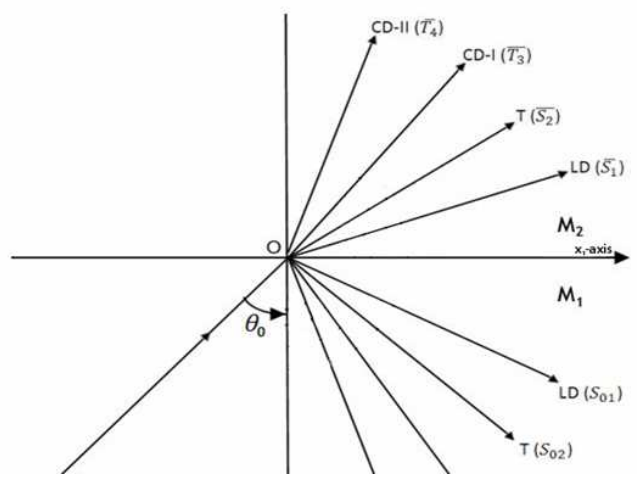

Figure 1: Geometry of the problem

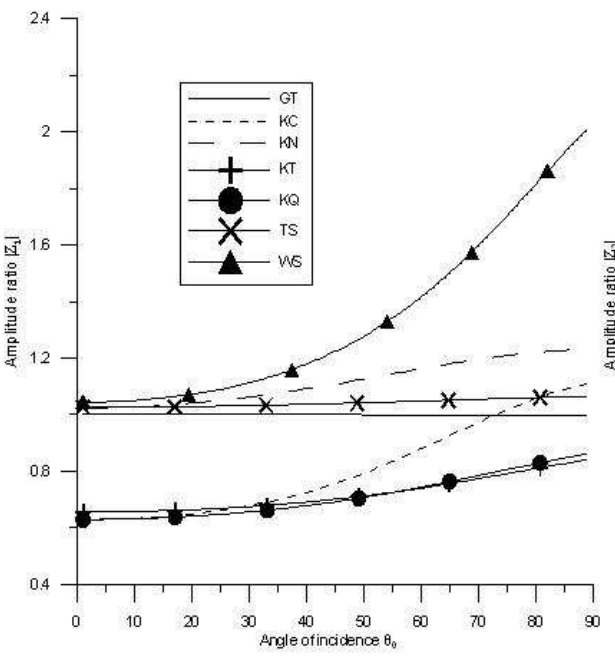

Fig.2

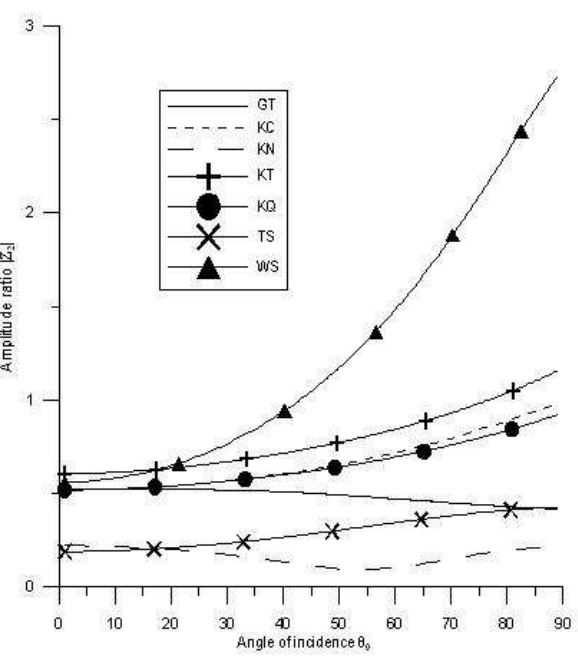

Fig.3 

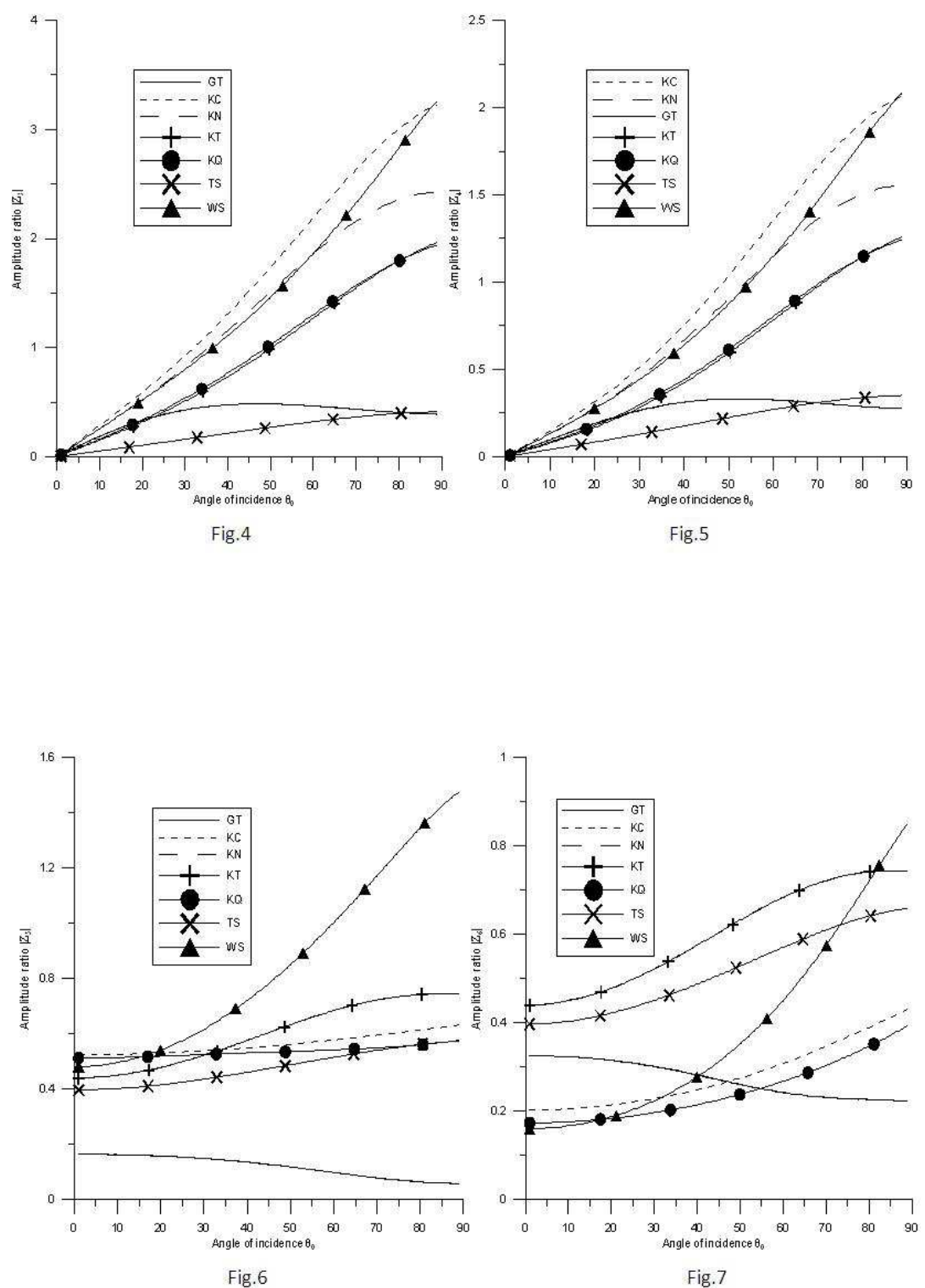

Fig. 6

Fig.7 


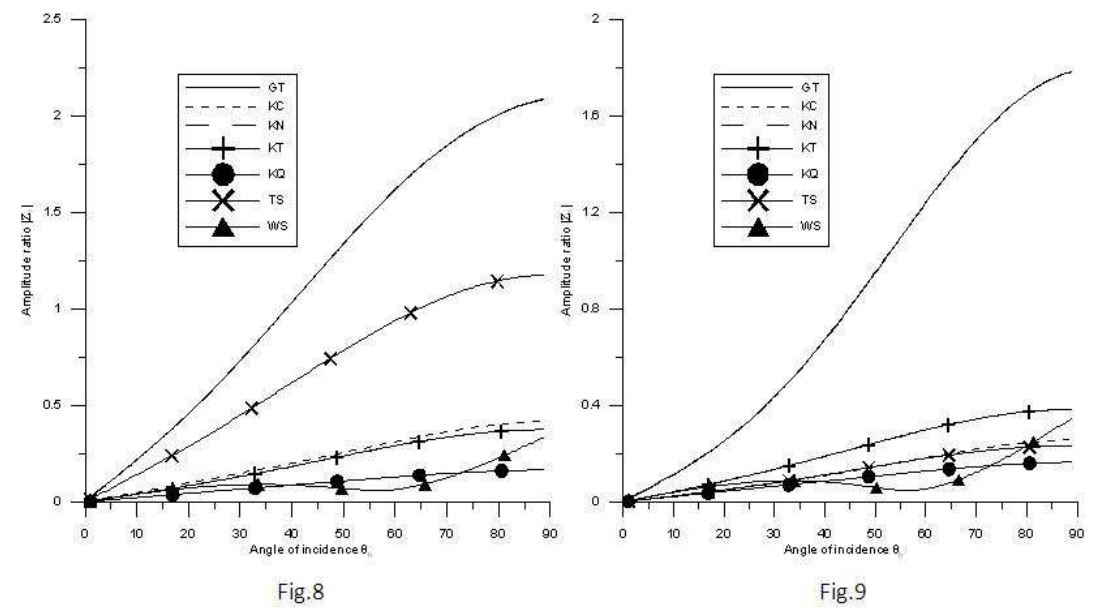

Figs. 2-9. Variations of amplitude ratios with the angle of incidence for LD-Wave

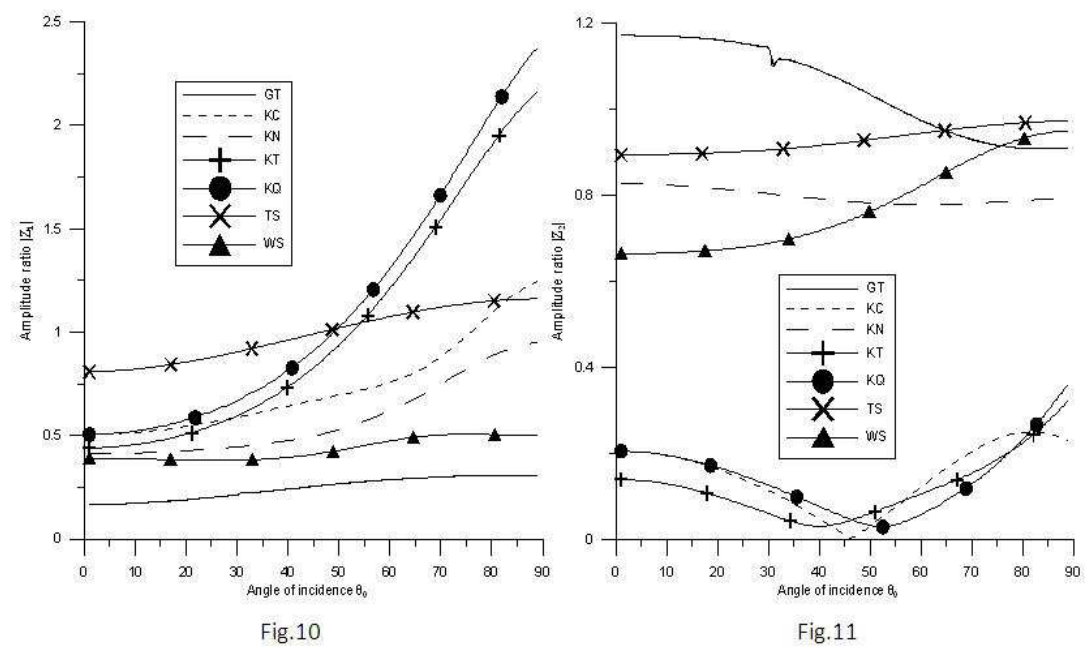



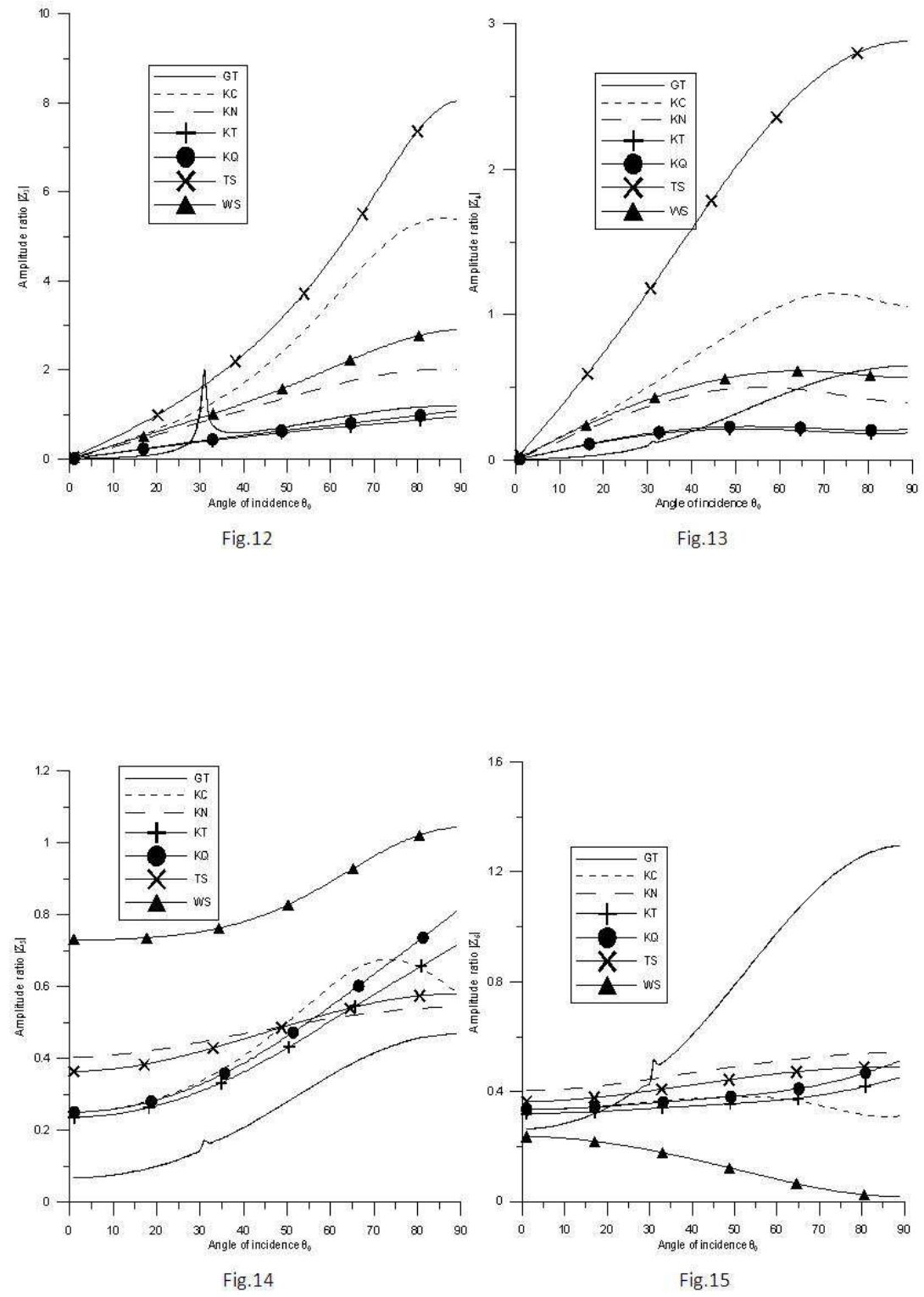


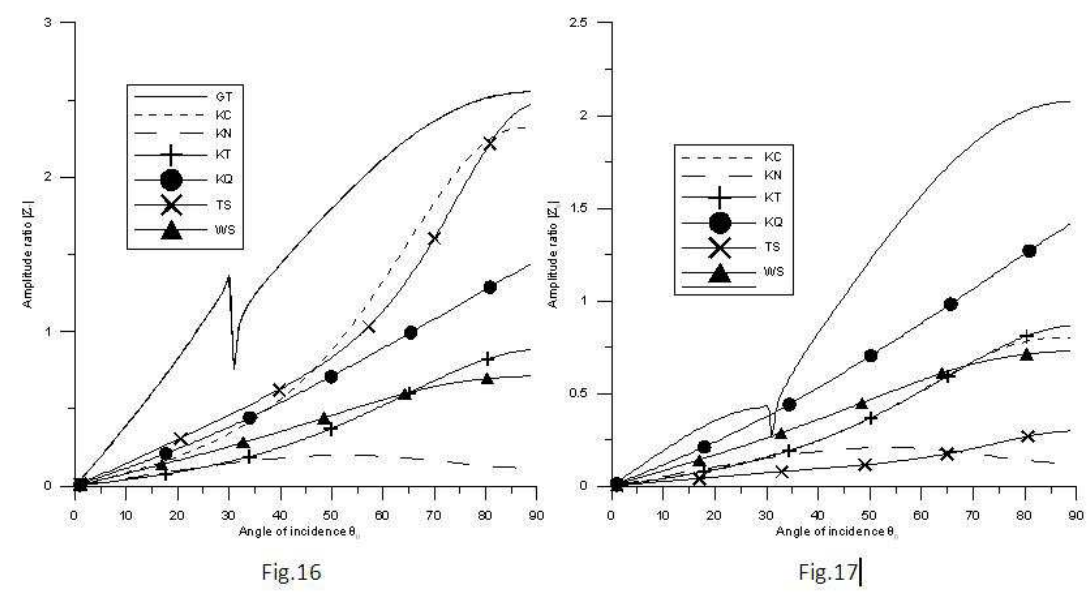

Figs. 10-17. Variations of amplitude ratios with the angle of incidence for T-Wave

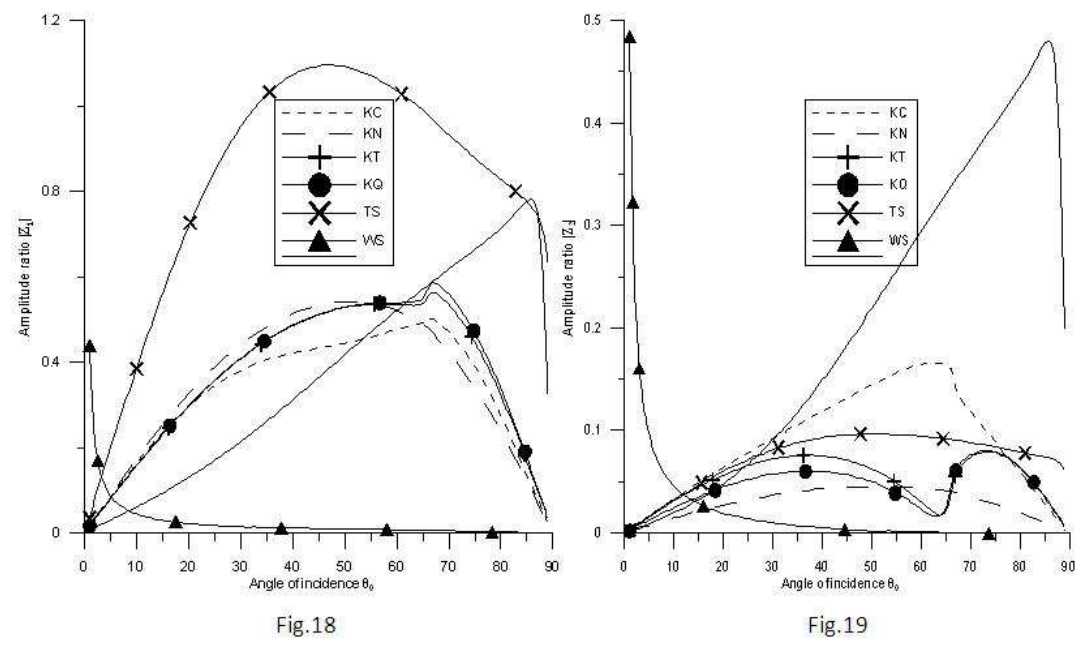



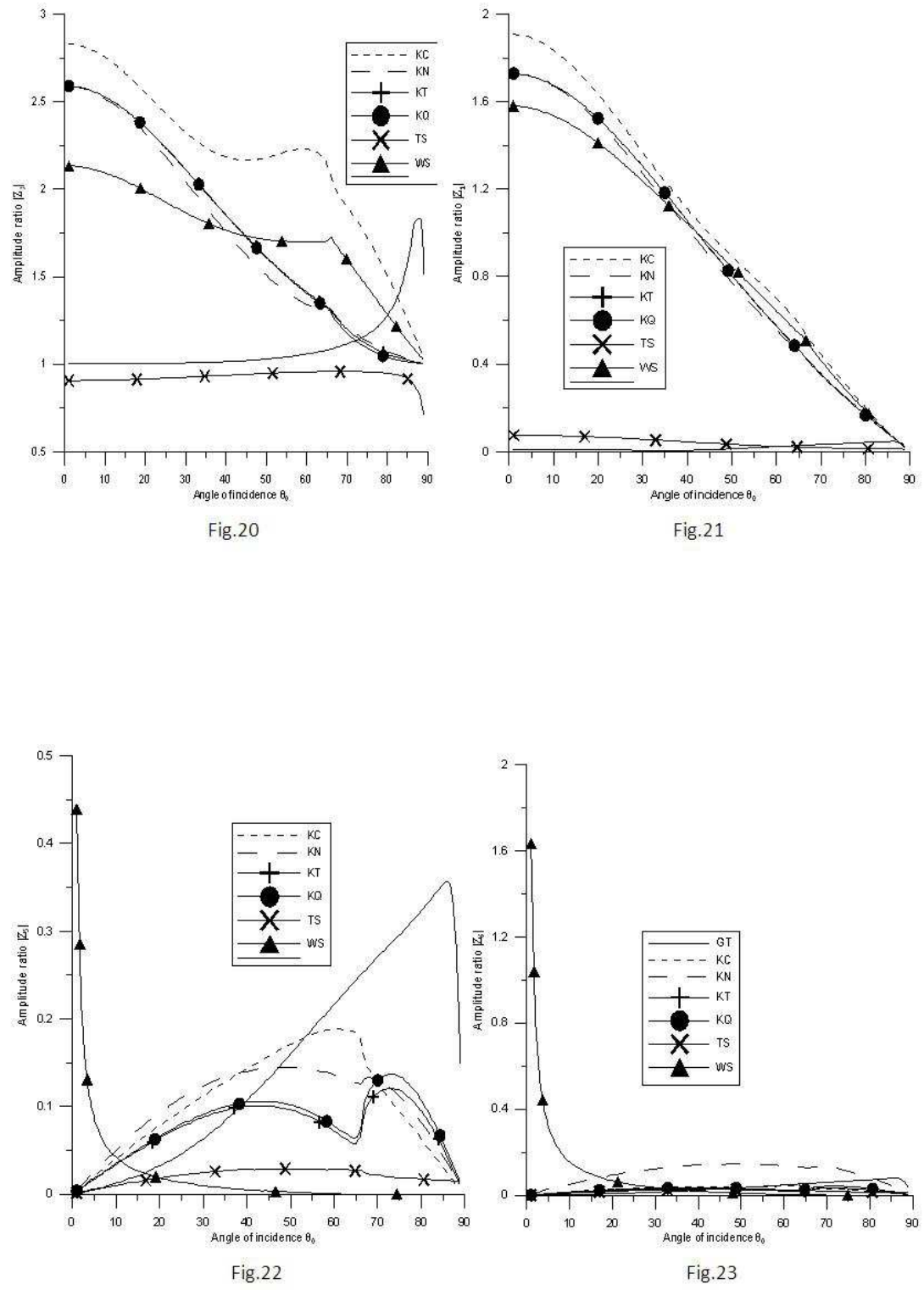


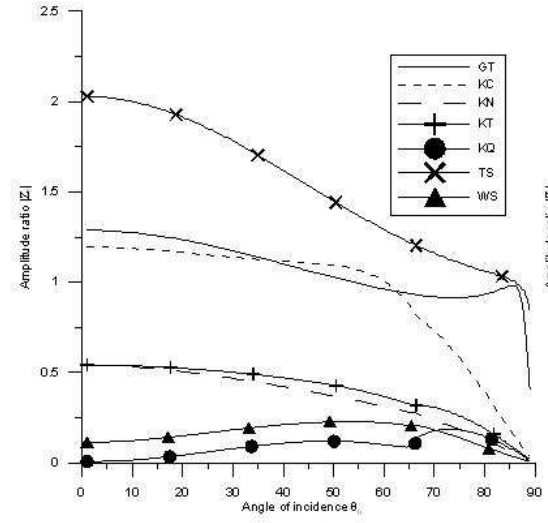

Fig.24

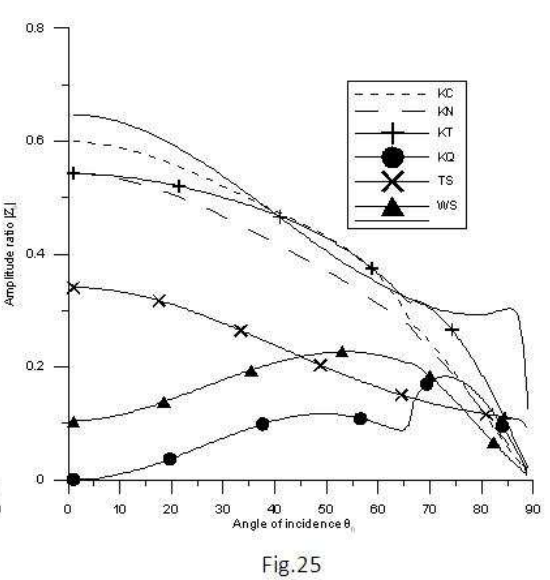

Fig. 25

Figs. 18-25. Variations of amplitude ratios with the angle of incidence for CD-I Wave

\section{References}

[1] A. C. Eringen, Linear theory of micropolar elasticity; J. Math. Mech. 15(1966a), 909-924.

[2] A. C. Eringen, 1966b Theory of micropolar fluids; J. Math.Mech. 16(1966b), 1-18.

[3] A. C. Eringen, Non-local polar field theories. In: Continuum Physics (ed.) A C Eringen, Vol.IV (New York,Academic Press) ,1976, 205-267.

[4] A.C. Eringen, Foundations of micropolar thermoelasticity, International Centre for Mechanical Science, Udline Course and Lectures 23, SpringerVerlag, Berlin, 1970.

[5] W. Nowacki, Theory of Asymmetric Elasticity-Oxford:Pergamon, 1986

[6] S. Dost and B. Taborrok, Generalized micropolar thermoelasticity, International Journal of Engineering Science, 16 (1978) 173-178.

[7] A.E. Green and K.A Lindsay, Thermoelasticity, Journal of Elasticity, 2 (1972) 1-7. 
[8] P.J. Chen, M.E. Gurtin and W.O. Williams, A note on non simple heat conduction, Zeitschrift fr angewandte Mathematik und Physik, 19 (1968) 960-970.

[9] P.J. Chen, M.E. Gurtin and W.O. Williams, On the thermoelastic material with two temperature, Zeitschrift fr angewandte Mathematik und Physik, 20 (1969) 107-112.

[10] M. Boley, Thermoelastic and irreversible thermodynamics, Journal of Applied Physics, 27(1956) 240-253.

[11] W.E. Warren and P.J. Chen, Wave propagation in the two temperature theory of thermoelasticity, Acta Mechanica, 16 (1973) 21-23.

[12] H.M. Youssef, Theory of two temperature generalized thermoelastic, IMA Journal of Applied Mathematics, (2005) 1-8.

[13] P. Puri and P. Jordan, On the propagation of harmonic plane waves under the two temperature theory, International Journal of Engineering Science, 44 (2006) 1113-1126.

[14] H.M. Youssef and E.A. Al-Lehaibi, A state approach of two temperature generalized thermoelasticity of one dimensional problem, International Journal of Solid and Structures, 44 (2007) 1550-1562.

[15] H.M. Youssef and H.A. Al-Harby, State space approach of two temperature generalized thermoelasticity of infinite body with a spherical cavity subjected to different types of thermal loading, Archive Applied Mechanics, 77 (2007) 675-687.

[16] A. Magana and R. Quintanilla, Uniqueness and growth of solution in two temperature generalized thermoelastic theories, Mathematics and $\mathrm{Me}$ chanics of Solids, Online (2008).

[17] S. Mukhopadhyay and R. Kumar, Thermoelastic interaction on two temperature generalized thermoelasticity in an infinite medium with a cylindrical cavity, Journal of Thermal Stresses, 32 (2009) 341-360.

[18] K. Roushan and M. Santwana, Effect of thermal relaxation time on plane wave propagation under two temperature thermoelasticity, International Journal of Engineering Science, 48 (2010) 128-139.

[19] S. Kaushal, N. Sharma and R. Kumar, Propagation of waves in generalized thermoelastic continua with two temperature, International Journal of Applied Mechanics and Engineering, 15 (2010) 1111-1127. 
[19] S. Kaushal, R. Kumar and A. Miglani, Wave propagation in temperature rate dependent thermoelasticity with two temperature, Mathematical Sciences, 5 (2011) 125-146.

[20] M.A. Ezzat and E.S. Aiwad, Constitutive relations, Uniqueness of solution and thermal shock application in the linear theory of micropolar generalized thermoelasticity involving two temperature, Journal of Thermal Stresses, 33 (2010) 226-250.

[21] M.A. Ezzat, F. Hamza and E. Awad, Electro Magneto-thermoelastic plane waves in micropolar solid involving two temperatures, Acta Mechanica Solida Sinica, 23 (2010) 200-212.

[23] J.M. Baik and R.B. Thomson, Ultrasonic scattering from imperfect interfaces a quasi-static model. Journal of Nondestructive Evaluation, 4 (1984) 177-176.

[22] S.I. Rokhlin , Adhesive joint characterization by ultrasonic surface and interface waves [M]- Adhesive joints: Formation, Characteristics and Testing. Edited by K.L. Mittal (plenum, New York), 1984, 307-345.

[23] T.C. Angel and J.D. Achenbach, Reflection and transmission of elastic waves by a periodic array of crack, Journal of Applied Mechanics, 52 (1985) 33-41.

[24] A. Pilarski and J.L. Rose, A transverse wave ultrasonic oblique- incidence technique for interface weakness detection in adhesive bonds, Journal of Applied Physics,63 (1988) 300-307.

[25] A.I. Lavrentyev and S.I. Rokhlin, Ultrasonic spectroscopy of imperfect contact interfaces between a layer and two solids, Journal of Acoustical Society of America, 103 (1998) 657-664.

[26] R. Kumar and N. Sharma, Effect of viscocity on wave propagation between two micropolar viscoelastic thermoelastic solids with two relaxation times having interfacial imperfections, International Journal of Manufacturing Science and Technology, 1 (2007) 133-152.

[27] R. Kumar, N. Sharma and P. Ram, Reflection and transmission of micropolar elastic waves at an imperfect boundary, Multidiscipline Modeling in Materials and Structure, 4 (2008) 15-36.

[28] R. Kumar, N. Sharma and P. Ram, Response of imperfections at the boundary surface, International eJournal of Engineering Mathematics:Theory and Applications (IeJEMTA), 3 (2008) 90-109. 
[29] R. Kumar, N. Sharma and P. Ram, Interfacial imperfection on reflection and transmission of plane waves in anisotropic micropolar media, Theoretical and Applied Fracture Mechanics, 49 (2008) 305-312.

[30] R. Kumar, N. Sharma and P. Ram, Effect of stiffness on reflection and transmission of micropolar thermoelastic waves at an interface between an elastic and micropolar generalized thermoelastic solid, Structural Engineering and Mechanics, an International Journal, 31 (2009) 117-135.

[31] P. Ram and N. Sharma, Reflection and Transmission of micropolar thermoelastic waves with an imperfect bonding, International Journal of Applied Mathematics and Mechanics, 4 (2008) 1-23.

[32] R. Kumar and N. Sharma, Effect of viscocity and stiffness on wave propagation in micropolar visoelastic media, International Journal of Applied Mechanics and Engineering, 4 (2009) 415-431.

[33] N. Sharma, S. Kaushal and R. Kumar, Effect of viscocity and stiffness on amplitude ratios in microstretch viscoelastic media, Applied Mathematics and Information Sciences, 5 (2011) 321-341.

[34] R. Kumar and V. Chawala, Effect of rotation and stiffness on surface wave propagation in a elastic layer lying over a generalized thermodiffusive elastic half space with imperfect boundary, Journal of Solid Mechanics, 2 (2010) 28-42.

[35] R. Kumar and V. Chawala, Effect of rotation on surface wave propagation in a elastic layer lying over a thermo diffusive elastic half space having imperfect boundary, International Journal of Applied Mechanics and Engineering, 16 (2011) 37-55.

[36] R. Kumar and V. Chawala, Wave propagation at the imperfect boundary between transversely isotropic thermodiffusive Eastic layer and half space, Journal of Engineering Physics and Thermophysics, 84 (2011)1192-1200.

[37] M. Marin, R.P. Agarwal, S.R. Mahmoud, Modeling a microstretch thermoelastic body with two temperature, Abstract and Applied Analysis, doi: 10.1155/2013/583464, Vol. 2013 (2031), 7 pg.

[38] M. Marin, A partition of energy in thermoelsticity of microstretch bodies, Nonlinear Analysis: RWA, Vol. 11, 4(2010), 2436-2447,

[39] M. Marin, Some estimates on vibrations in thermoelasticity of dipolar bodies, Journal of Vibration and Control, Vol. 16, 1(2010), 33-47 
[40] M. Marin, An evolutionary equation in thermoelasticity of dipolar bodies, Journal of Mathematical Physiscs, Vol. 40, 3(1999), 1391-1399

Kunal SHARMA,

Department of Mechanical Engineering,

NIT Kurukshetra,

Kurukshetra 136119, India

Email: kunal_nit90@rediffmail.com

Marin MARIN,

Department of Mathematics and Computer Sciences,

Transilvania University of Brasov,

Bdul Iuliu Maniu, nr. 50, Brasov, Romania.

Email: m.marin@unitbv.ro 
